\title{
Clinical Prediction Score for Early Neuroimaging in Acquired Isolated Oculomotor Nerve Palsy
}

This article was published in the following Dove Press journal: Eye and Brain

Juthamat Witthayaweerasak (D) Natchada Tansuebchueasai (D) Nipat Aui-aree

Department of Ophthalmology, Faculty of Medicine, Prince of Songkla University, Hatyai, Songkhla, Thailand
Correspondence: Juthamat

Witthayaweerasak

Department of Ophthalmology, Faculty of Medicine, Prince of Songkla University, 15

Kanjanavanit Road, Hatyai, Songkhla

90110 , Thailand

Tel +66-8I-599-2099

Fax +66-7442-9619

Email juthamat.w@psu.ac.th
Purpose: An immediate neuroimaging investigation in patients with isolated oculomotor nerve palsy (ONP) remains controversial. We aimed to develop a clinical prediction score to determine whether or not acquired isolated ONP patients require prompt imaging.

Methods: A retrospective study was performed. Demographic data and clinical presentations were collected to determine predictive factors favored for early brain imaging using multivariate logistic regression analysis.

Results: Ninety-seven eyes of 96 patients diagnosed with isolated ONP were included. Forty-one eyes (42.3\%) were caused by ischemia, while the other 56 eyes $(57.7 \%)$ were caused by non-ischemic etiologies, namely aneurysm $(n=22)$, trauma $(n=18)$, inflammation $(\mathrm{n}=5)$, tumor $(\mathrm{n}=4)$, and others $(\mathrm{n}=7)$. Eighty-two eyes $(84.5 \%)$ had undergone neuroimaging study due to initially suspected non-ischemic causes. Only $36(43.9 \%)$ revealed concordant diagnosis. The potential clinical predictors favored for neuroimaging using multivariate logistic regression analysis were age 10-50 years (adjusted odds ratio [aOR] 9.01, 95\% CI: 1.25-64.8), age 51-70 years (aOR 1.71, 95\% CI: 0.46-6.35), history of head trauma (aOR 7.14, 95\% CI: 1.19-42.9), absence of vascular risk factors (aOR 3.85, 95\% CI: 1.23-12.1), and poor pupillary response (aOR 6.96, 95\% CI: 1.99-24.3). The predictor scores with an area under the ROC curve of 0.852 were 4, 1, 4, 3, and 4, respectively. The optimum cut-point was 3 for a sensitivity of $96 \%$ and specificity of $44 \%$.

Conclusion: Patients with acquired isolated ONP should be considered early neuroimaging studies when they are younger than 50 years old, have a history of head trauma, have no history of vascular risk factors, or have poor pupillary reaction.

Keywords: third nerve palsy, oculomotor nerve palsy, predictor, neuroimaging

\section{Introduction}

Isolated oculomotor nerve palsy (ONP) is quite common in general practice. Various causes of ONP have been described in several studies, for instance ischemia, compression from aneurysm or tumor, and trauma. ${ }^{1-4}$ Ischemia was the most common etiology with spontaneous recovery, and it was presumed based on some helpful clinical clues before imaging became available. ${ }^{2,3,5,6}$ Nowadays, high quality neuroimaging studies, such as computed tomography (CT), magnetic resonance imaging (MRI), magnetic resonance angiography (MRA), and angiogram, have played a more important role aiding the diagnosis. Consequently, more serious but treatable causes were identified including intracranial aneurysm, tumor, inflammation, infection, and brain stem infarction. ${ }^{7-10}$ However, controversy remains as to which patients require an immediate neuroimaging study. $^{9-15}$ The objective of this study was therefore to determine the predictive factors of clinical characteristics of acquired isolated ONP patients for an early neuroimaging study. 


\section{Materials and Methods}

\section{Patients}

This was a retrospective review of all acquired isolated ONP patients who visited neuro-ophthalmology, neuromedicine, and neurosurgery clinics at Songklanagarind Hospital, Prince of Songkla University, Songkhla, Thailand from July 1, 2003 to April 30, 2017. Ethics approval was obtained from The Human Research Ethics Committee, Faculty of Medicine, Prince of Songkla University. The ethics committee determined that written consent was not required as risk of this research was not greater than minimal risk and the waiver will not adversely affect the rights and welfare of study participants. The patient data was also maintained with confidentiality and in compliance with the Declaration of Helsinki. The patients were identified according to the diagnostic criteria of third (oculomotor) nerve palsy by ICD-10 (H49.0). The excluded patients had associated cranial nerve II, IV, V, VI, or VII involvement, congenital ONP, and other causes of diplopia and ptosis, eg, ocular myasthenia gravis and thyroid-associated orbitopathy. Ninety-seven eyes of 96 patients with acquired isolated ONP were included. Since the diagnosis was based on the clinical presentation, neuroimaging investigations were not a prerequisite. The patients were classified into two groups according to the etiology: ischemic or non-ischemic. The patients with clinical characteristics suggestive of an ischemic cause, eg, $>50$ years old, underlying atherosclerotic disease, no pupillary involvement, and negative neuroimaging study results (if present) were categorized into the ischemic group. ${ }^{9-11}$ The non-ischemic group included other causes, ie, aneurysm, trauma, tumor, inflammation, and others. Traumatic ONP was defined as immediate onset after head trauma. Neuroimaging investigations; for example, computed tomography (CT), magnetic resonance imaging (MRI), computed tomographic angiography (CTA), magnetic resonance angiography (MRA), digital subtraction angiogram and their diagnostic concordance were also collected.

\section{Clinical Predictors}

Demographic data, clinical manifestations, including ocular and neurological symptoms, underlying diseases, current aspirin use, history of smoking, initial eye examinations including best-corrected visual acuity (BCVA, measured by $\log$ MAR or the log of the minimum angle of resolution), pupillary involvement, motility deficits, and ptosis parameters were collected and analyzed to identify significant correlations between these parameters and according to the etiology. The coexisting common vascular risk factors were defined as present if the patent had an underlying disease such as diabetes mellitus, hypertension, dyslipidemia, coronary artery disease, or stroke defined by the diagnosis of an internist or according to the current medications. Based on the presented scenario, we categorized patients into three groups: 1) complete or incomplete ONP with pupillary involvement; 2) incomplete ONP with pupil sparing; and 3) complete ONP with pupil sparing. Pupillary involvement was defined as one of the following: presence of anisocoria $>1 \mathrm{~mm}$ or sluggish or no pupillary response. ${ }^{6,16}$ Complete motility deficit was defined as $100 \%$ limitation of eye movement. ${ }^{8}$ Complete ptosis was defined as complete weakness of the levator palpebrae superioris muscle. ${ }^{9,11}$

\section{Statistical Analysis}

R (R Core Team, 2019) was used for all analyses. ${ }^{17}$ We used a multiple imputation method to impute missing data. To compare between the two groups, Student's $t$-test, Pearson's chi-square test or Fisher's exact test was used. The initial multivariate analysis included variables with $p$ values $<0.2$ from the univariate analysis. The potential predictive factors for non-ischemic cause were then analyzed by multivariate logistic regression analysis and are shown as adjusted odds ratios which were significant when the $95 \%$ confidence intervals did not include a value of 1 . The coefficients from the model were multiplied by two and rounded to the nearest integer to create a prediction score.

\section{Results}

\section{Baseline Characteristics}

Ninety-seven eyes of 96 patients (48 males and 48 females) were included in this study. The mean age of all patients at diagnosis was $57.6 \pm 16.1$ years (median 59.1 years, range $10.7-93.6$ years). Ninety-five patients (98.9\%) were affected unilaterally. Forty-one eyes (42.3\%) were caused by ischemia while the other $56(57.7 \%)$ were caused by non-ischemic etiologies including aneurysm $(n=22)$, head trauma $(n=18)$, inflammation $(\mathrm{n}=5)$, tumor $(\mathrm{n}=4)$, postoperative intracranial surgery $(\mathrm{n}=3)$, subdural hematoma causing uncal herniation $(\mathrm{n}=1)$, stroke of midbrain $(\mathrm{n}=1)$, and undetermined cause $(n=2)$. The aneurysm causes included posterior communicating artery aneurysm $(\mathrm{n}=21)$ and right cavernous 
internal carotid artery (ICA) aneurysm $(n=1)$. The tumor causes included cavernoma of the midbrain $(n=1)$, cavernous meningioma $(n=1)$, brain metastasis $(n=1)$, and sellar chordoma $(n=1)$. The inflammation causes included neuritis $(\mathrm{n}=2)$, pachymeningitis $(\mathrm{n}=2)$, and post-viral infection with history of preceding upper respiratory tract infection $(n=1)$.

Table 1 shows the characteristics of the 41 eyes in the ischemic group and the 56 eyes in the non-ischemic group. Age was significantly different between the two groups. Patients with ischemic ONP were predominantly older ( $>50$ years old). From the initial evaluation, history of ptosis and head trauma, underlying diseases associated with vascular risk factors, and pupil examination including pupil size, anisocoria, and reaction to light were statistically different between the two groups. In contrast, BCVA, the duration of diplopia and ptosis, degree of motility deficit, and ptosis were similar in both groups. Based on clinical presentation: 55 patients had complete or incomplete ONP with pupillary involvement, 15 had incomplete ONP with pupil sparing, and 10 had complete ONP with pupil sparing. In the pupillary involvement group, 17 of 55 eyes $(30.9 \%)$ had ONP caused by ischemia. In the pupil sparing group, 6 of 15 eyes (40\%) had incomplete ONP and 3 of 10 eyes $(30 \%)$ had complete ONP caused by nonischemia.

\section{Clinical Predictors}

Table 2 presents the predictors for non-ischemic ONP based on the multivariate logistic regression model. The only significant factors were age group, history of trauma, absence of vascular risk factors, and pupillary reaction. The receiver-operator characteristic curve for the final logistic regression model in Figure 1 showed an area under the curve of 0.852 .

The scores for each predictor of age 10-50 years, age 51-70 years, history of head trauma, absence of vascular risk factors, and sluggish/no pupillary response were 4,1 , 4,3 , and 4, respectively, otherwise the score was 0 (Table 2). Summation of each predictor score resulted in a final predictive score for each patient. The resultant mean (SD) score was 6.4 (4.3) (range 0-15). The optimal cut-point that maximized the sensitivity and specificity was 7.0. However, this cut point resulted in a sensitivity of only $66 \%$ and a specificity of $88 \%$. In order to increase the sensitivity, we chose a cut-point of 3.0 to give a sensitivity of $96 \%$ while the specificity decreased to $44 \%$.

\section{Neuroimaging}

At the initial presentation, 82 eyes $(84.5 \%)$ underwent or were scheduled for a neuroimaging study due to suspected non-ischemic cause. The neuroimaging investigations consisted of MRI (49 eyes), CT (43 eyes), MRA (34 eyes), CTA (13 eyes) and digital subtraction angiogram (35 eyes). Only 36 eyes (43.9\%) revealed a concordant diagnosis. The intracranial aneurysms were detected on CTA and MRA (Figure 2A) followed by angiogram (Figure 2B) in 4 , and 8 eyes, and on CT or MRI followed by angiogram in 10 eyes, respectively. Other etiologies of ONP included, trauma, tumor and inflammation, which were evaluated by MRI in 16 eyes and CT in 15 eyes. Among the 46 eyes with a discordant diagnosis, 11, which were initially diagnosed as non-ischemia, due mostly to pupillary involvement, were later confirmed as ischemia. Fifteen of 97 eyes (15.5\%) did not have initial imaging, of which 4 underwent neuroimaging (MRI with CTA or MRA) due to clinical worsening during the patient's follow-up visits. In these patients ONP was caused by ischemia.

\section{Discussion}

In our study, ischemia was the main cause of ONP, followed by aneurysm, head trauma, tumor, and inflammation. These results were similar to another populationbased study, but the presumed microvascular cause was followed by trauma, compression from neoplasm, postneurosurgery, and lastly compression from aneurysm. ${ }^{18}$ In contrast to another study conducted in Thailand, trauma was the major cause followed by ischemia. ${ }^{19}$

It is known that clinical clues can be helpful to determine the cause of isolated ONP. Similar to previous studies, $30.9 \%$ of pupil-involved ONP was caused by ischemia. $^{13,20,21}$ This might lead to an overuse of the investigation. While Chou et al found that up to $50 \%$ of pupil-spared patients with incomplete motility deficit were caused by non-ischemia, we found a lower prevalence of $40 \%$ with incomplete and $30 \%$ with complete motility deficit. ${ }^{13}$ These patients might need an immediate imaging study. Therefore, sparing of the pupil alone cannot definitely differentiate a non-ischemic cause. Delay in treating some causes, such as aneurysm, can be fatal. Early neuroimaging can be useful, though routine use remains impractical. Therefore, defining the right etiology at presentation is problematic. 
Table I Comparison of Demographic Data and Clinical Manifestations in Ischemic and Non-Ischemic Oculomotor Nerve Palsy

\begin{tabular}{|c|c|c|c|}
\hline Characteristic & Ischemic $(n=41)$ & Non-Ischemic $(n=56)$ & $P$ value \\
\hline Age group (years) & & & $<0.001^{\mathrm{a}}$ \\
\hline $10-50$ & $3(7.3)$ & $23(4 I . I)$ & \\
\hline $5 \mathrm{I}-70$ & $24(58.5)$ & $27(48.2)$ & \\
\hline $7 I-100$ & $14(34.1)$ & $6(10.7)$ & \\
\hline Gender & & & 0.062 \\
\hline Male & $25(62.5)$ & $23(4 I .1)$ & \\
\hline Female & $15(37.5)$ & $33(58.9)$ & \\
\hline Diplopia & $28(68.3)$ & $29(51.8)$ & 0.155 \\
\hline Duration of diplopia (days), mean (SD) & $32.6(79.3)$ & $4 \mid 2.0(\mid 744.7)$ & 0.273 \\
\hline Ptosis & $39(95.1)$ & $43(76.8)$ & $0.029^{\mathrm{a}}$ \\
\hline Duration of ptosis (days), mean (SD) & $21.9(60.2)$ & $57.5(123.3)$ & 0.109 \\
\hline Eye pain & $14(34.1)$ & $13(23.2)$ & 0.338 \\
\hline Headache & $23(56.1)$ & $32(57.1)$ & I \\
\hline Previous head trauma & $2(4.9)$ & $18(32.1)$ & $0.002^{\mathrm{a}}$ \\
\hline Vascular risk factors (any) & $34(82.9)$ & $18(32.1)$ & $<0.001^{\mathrm{a}}$ \\
\hline Stroke & $8(19.5)$ & $\mathrm{I}(\mathrm{l} .8)$ & $0.004^{\mathrm{a}}$ \\
\hline Diabetes mellitus & $18(43.9)$ & $6(10.7)$ & $<0.001^{\mathrm{a}}$ \\
\hline Hypertension & $27(65.9)$ & $16(28.6)$ & $<0.001^{\mathrm{a}}$ \\
\hline Dyslipidemia & $14(34.1)$ & $4(7.1)$ & $0.002^{\mathrm{a}}$ \\
\hline Coronary artery disease & $4(9.8)$ & $2(3.6)$ & $0.238^{\mathrm{a}}$ \\
\hline Currently taking aspirin & $8(19.5)$ & $4(7.1)$ & 0.13 \\
\hline Smoking & II (26.8) & $16(28.6)$ & I \\
\hline BCVA (logMAR), mean (SD) & $0.5(0.5)$ & $0.4(0.4)$ & 0.209 \\
\hline Ptosis & $36(97.3)$ & 44 (91.7) & 0.382 \\
\hline Eye motility deficit & $38(100)$ & $47(94)$ & 0.255 \\
\hline Pupil size in affected eye $(\mathrm{mm})$, mean $(\mathrm{SD})$ & $3.7(1.1)$ & $5.0(1.5)$ & $<0.001^{\mathrm{a}}$ \\
\hline Anisocoria (mm), mean (SD) & $1.0(1.0)$ & $2.3(1.5)$ & $<0.001^{\mathrm{a}}$ \\
\hline Pupillary reaction & & & $<0.001^{\mathrm{a}}$ \\
\hline Normal reaction & $21(58.3)$ & $9(18)$ & \\
\hline Sluggish/no response & $15(4 \mid .7)$ & $41(82)$ & \\
\hline Clinical presentation & & & $0.016^{\mathrm{a}}$ \\
\hline Complete/incomplete ONP with pupillary involvement & 17 & 38 & \\
\hline Incomplete ONP with pupil sparing & 9 & 6 & \\
\hline Complete ONP with pupil sparing & 7 & 3 & \\
\hline
\end{tabular}

Notes: Data are presented as $\mathrm{n}(\%)$ unless indicated otherwise. ${ }^{a} \mathrm{p}$ value $<0.05$.

Abbreviations: ONP, oculomotor nerve palsy; BCVA, best-corrected visual acuity; SD, standard deviation.

Our study described the clinical characteristics that distinguish patients with ischemic ONP from non-ischemic ONP. Various studies have found an association between the patient's age and risk of ischemia. ${ }^{13-15}$ Likewise, we found that the majority of ischemic patients were $>50$ years old. However, in the 10-50 years age group, history of head trauma, absence of vascular risk factors, and a sluggish or non-reactive pupil to light were found to be parameters 
Table 2 Final Multivariate Logistic Regression Predicting Non-Ischemia and Scoring System

\begin{tabular}{|l|l|l|l|l|l|}
\hline Factors & aOR $(\mathbf{9 5} \% \mathbf{~ C l})$ & P value (Wald's test) & P value (LR-test) & Coefficient & Score \\
\hline Age group: $>$ 70 years & $\mathrm{I}$ & & 0.06 & - & 0 \\
$51-70$ & $\mathrm{I} .71(0.46-6.35)$ & 0.42 & & 0.54 & 1 \\
I0-50 & $9.01(1.25-64.8)$ & 0.03 & & 2.20 & 4 \\
Trauma: no & $\mathrm{I}$ & & 0.015 & - & 0 \\
Yes & $7.14(1.19-42.9)$ & 0.03 & 0.018 & 1.97 & 4 \\
Vascular risk factors: yes & $\mathrm{I}$ & & & - & 0 \\
No & $3.85(1.23-12.1)$ & 0.02 & $<0.001$ & 1.35 & 3 \\
Pupillary reaction: normal & $\mathrm{I}$ & & & - & 0 \\
Sluggish/no reaction & $6.96(1.99-24.3)$ & 0.002 & 1.94 & 4 \\
\hline
\end{tabular}

Abbreviations: aOR, adjusted odds ratio; $\mathrm{Cl}$, confidence interval; $\mathrm{LR}$, likelihood ratio.

associated with non-ischemia and therefore patients with these indicators should be considered for initial neuroimaging studies. In this study, the scoring system using an appropriate cutpoint was determined to give high sensitivity so that the nonischemic cause, especially aneurysm, was less likely to be missed. Patients with a score $>3.0$ should be suspected of having non-ischemia. With a sensitivity of $96 \%$, the two misclassified patients in the ischemia group included brain metastasis and ICA aneurysm. Both patients presented with progressive ONP with good pupillary response for the past several months and had underlying diseases such as lung cancer, dyslipidemia, hypertension, and coronary artery disease. We considered these patients as an outlier who had unusual clinical manifestations with a clue suggestive of compression more than ischemia.

Similar to our scoring system, it is widely accepted that early neuroimaging be performed in acquired isolated ONP patients who are $<50$ years old. ${ }^{6}$ Among those $>50$ years old, the role of neuroimaging is still controversial. ${ }^{9-14}$ Two studies in acute isolated ocular mononeuropathies showed opposite results. ${ }^{13,15}$ Both studies included patients who were $>50$ years old with no history of cancer, neurological or orbital disease, or history of head trauma. Focusing on ONP only, Chou et al found a 13.8\% (4/29) incidence of other identifiable etiologies which were comprised of neoplasm $(n=1)$, brain stem infarction $(n=1)$, and posterior communicating $(n=2) .{ }^{13}$ Considering the significance of the finding, the author favored performing neuroimaging in all patients. On the other hand, Murchinson found no lesions from MRI in 14 patients with ONP and concluded that it was not cost-effective to perform MRI in all patients. ${ }^{15}$ Unlike those two studies, we included patients in all age groups and also those who had a neurological condition and a history of cancer or trauma to demonstrate the overall prevalence. Since our study considered the association with other factors to calculate a final score, making a decision to perform initial neuroimaging is easier.

The presence or absence of vascular risk factors was considered in the scoring system. Several previous studies reported strong associations between vascular risk factors and ischemic etiology but failed to demonstrate any significant individual factors and microvascular etiology. 6,13,14,21 Trends were seen for diabetes and hypertension only. We found that up to $32.1 \%$ of patients in the non-ischemic group also had at least one vascular risk factor. This rate was lower than reported by Chou et al (50\%) and Tamhankar et al $(60 \%)$ which can be explained by their inclusion of patients aged $\geq 50$ years. ${ }^{13,14}$

Tamhankar et al found that up to $60 \%$ of patients with isolated ONP experienced pain or headache, while

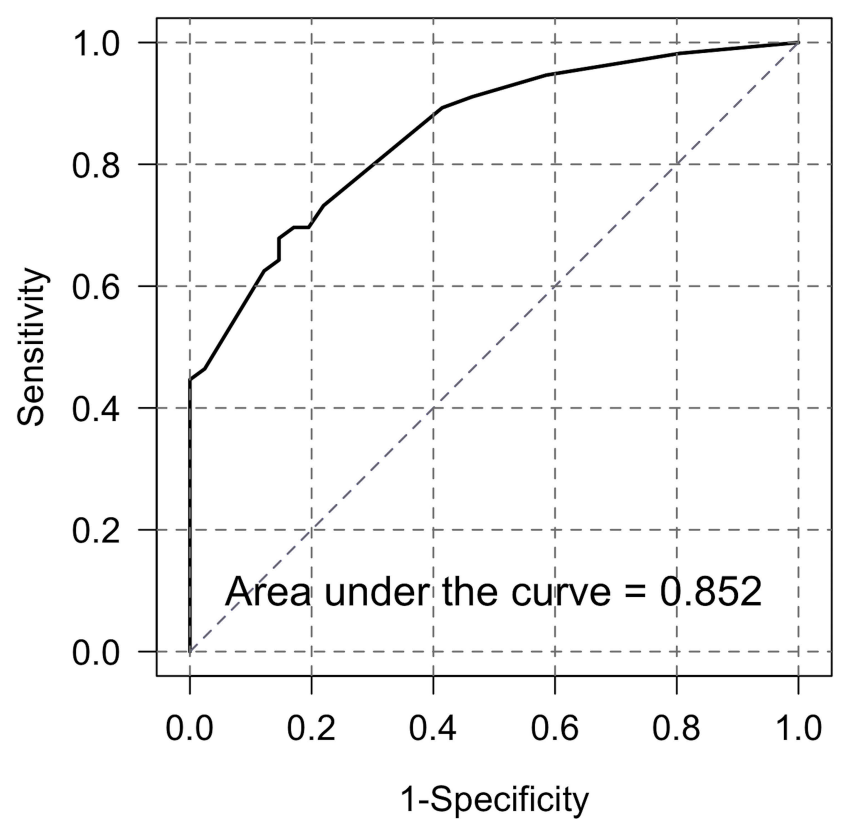

Figure I Receiver operator characteristic curve for the final logistic regression model. 


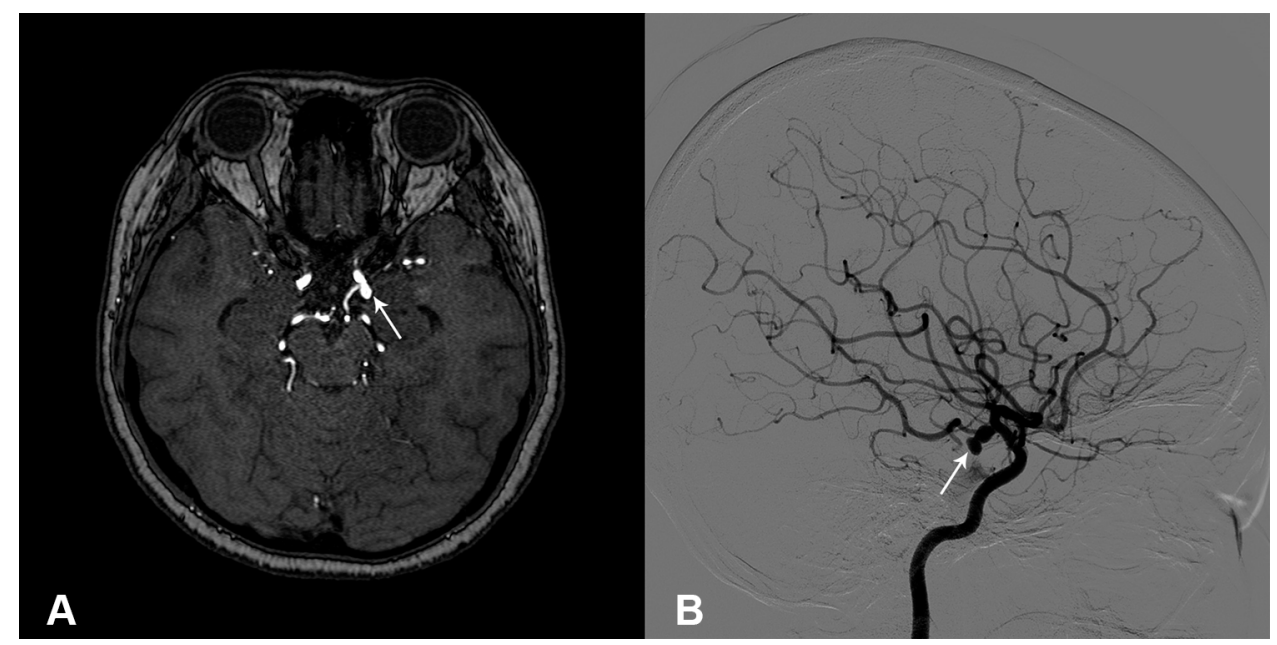

Figure 2 (A) MRA demonstrating a left posterior communicating artery aneurysm (arrow), (B) Digital subtraction angiogram demonstrating a lobulated aneurysm (arrow) at left posterior communicating artery origin.

our results revealed that $27.8 \%$ of patients had eye pain and $56.7 \%$ had headache. ${ }^{14}$ Both eye pain and headache are common symptoms and can be reported in both ischemia and aneurysm making it impossible to distinguish these two etiologies, similar to our study. ${ }^{9,10,14,21}$

In the past, we evaluated patients with ONP, and determined the etiologies from clinical clues. ${ }^{13,15}$ Nowadays, modern and non-invasive neuroimaging studies have been widely available and accepted. ${ }^{11,22,23}$ In our study, the rate of performing neuroimaging was high, which was similar to previous studies. $5,11,12,14,22$ We also demonstrated the usefulness of CTA or MRA, to detect intracranial aneurysms was comparable to conventional angiograms. It is by now generally accepted that both CTA and MRA have high accuracy rates for detection of aneurysms larger than $3 \mathrm{~mm} .^{22,24-27}$ Additionally, the cost is relatively low compared with angiograms. ${ }^{24,26}$ However, digital subtraction angiography is the gold standard to diagnose intracranial aneurysm, previously, it also has a low potential risk of neurologic complications. ${ }^{22,24-28}$

If we performed neuroimaging in all patients suspected of non-ischemic cause from the scoring system with a cut point of $3.0,79.4 \%$ would have undergone a neuroimaging study at initial presentation with $70.1 \%$ having a concordant diagnosis. Compared to what our results revealed (84.5\% with initial neuroimaging study and $43.9 \%$ with concordant diagnosis), the frequency of performing a neuroimaging study is lower while the rate of concordance is much higher.

Since our study was conducted retrospectively, some patients were lost to follow-up and therefore we had missing data. We compensated this problem by using a multiple imputation method. Also, the patients in our study were recruited from a tertiary referral center, which can result in a selection bias. The referring physicians might have clinical clues that led them to seek additional consultation and therefore more serious etiologies might have been identified. Some physicians might refer the patients because the initial neuroimaging results revealed an underlying lesion. Therefore, these results might not reflect population-based data.

\section{Conclusion}

The scoring system developed from this study should be used to consider early neuroimaging to bridge the gap between the patient's expectations from investigations and the doctor's fear of missing treatable etiologies. With the high sensitivity of $96 \%$, the non-ischemic cause, especially aneurysm, is less likely to be missed. Also, the predictive score is practical and requires only a medical history and fundamental eye examination obtained by a general practitioner. Patients with a score $<3.0$ should be closely observed to prevent performing unnecessary neuroimaging.

\section{Acknowledgment}

The authors acknowledge with appreciation Mr. Edward McNeil from Faculty of Medicine, Prince of Songkla University for statistical analysis.

\section{Disclosure}

The authors report no conflicts of interest in this work. 


\section{References}

1. Berlit P. Isolated and combined pareses of cranial nerves III, IV and VI a retrospective study of 412 patients. J Neurol Sci. 1991;103 (1):10-15. doi:10.1016/0022-510X(91)90276-D

2. Richards BW, Jones FR, Younge BR. Causes and prognosis in 4278 cases of paralysis of the oculomotor, trochlear, and abducens cranial nerves. Am J Ophthalmol. 1992;113(5):489-496. doi:10.1016/S00029394(14)74718-X

3. Ramella MG, Muci-Mendoza R. Ischemic-microvascular ocular motor nerve palsies: a 7-year experience of the neuro-ophthalmology unit, Caracas Vargas Hospital. Int Congr Ser. 2002;1237:195-205. doi:10.1016/S0531-5131(01)00586-6

4. Keane JR. Third nerve palsy: analysis of 1400 personally-examined inpatients. Can J Neurol Sci. 2010;37(5):662-670. doi:10.1017/ S0317167100010866

5. Tamhankar MA, Volpe NJ. Management of acute cranial nerve 3, 4 and 6 palsies: role of neuroimaging. Curr Opin Ophthalmol. 2015;26 (6):464-468. doi:10.1097/ICU.0000000000000200

6. Jacobson DM. Risk factors for ischemic ocular motor nerve palsies. Arch Ophthalmol. 1994;112(7):961. doi:10.1001/archopht.1994.0109 0190109029

7. Blake PY, Mark AS, Kattah J, Kolsky M. MR of oculomotor nerve palsy. AJNR Am J Neuroradiol. 1995;16(8):1665-1672.

8. Jacobson DM, Trobe JD. The emerging role of magnetic resonance angiography in the management of patients with third cranial nerve palsy. Am J Ophthalmol. 1999;128(1):94-96. doi:10.1016/S00029394(99)00107-5

9. Lee AG, Hayman LA, Brazis PW. The evaluation of isolated third nerve palsy revisited. Surv Ophthalmol. 2002;47(2):137-157. doi:10.1016/S0039-6257(01)00303-4

10. Kung N, Van Stavern G. Isolated ocular motor nerve palsies. Semin Neurol. 2015;35(05):539-548. doi:10.1055/s-0035-1563568

11. Schultz KL, Lee AG. Diagnostic yield of the evaluation of isolated third nerve palsy in adults. Can J Ophthalmol. 2007;42(1):110-115. doi:10.3129/canjophthalmol.06-099

12. Volpe NJ, Lee AG. Do patients with neurologically isolated ocular motor cranial nerve palsies require prompt neuroimaging? J NeuroOphthalmol. 2014;34(3):301-305. doi:10.1097। WNO.0000000000000149

13. Chou KL, Galetta SL, Liu GT, et al. Acute ocular motor mononeuropathies: prospective study of the roles of neuroimaging and clinical assessment. J Neurol Sci. 2004;219(1-2):35-39. doi:10.1016/j. jns.2003.12.003

14. Tamhankar MA, Biousse V, Ying G-S, et al. Isolated third, fourth, and sixth cranial nerve palsies from presumed microvascular versus other causes. Ophthalmology. 2013;120(11):2264-2269. doi:10.1016/ j.ophtha.2013.04.009
15. Murchison AP. Neuroimaging and acute ocular motor mononeuropathies. Arch Ophthalmol. 2011;129(3):301. doi:10.1001/ archophthalmol.2011.25

16. Trobe JD. Managing oculomotor nerve palsy. Arch Ophthalmol. 1998;116(6):798. doi:10.1001/archopht.116.6.798

17. $\mathrm{R}$ Core Team 2019. A language and environment for statistical computing. $R$ Found Stat Comput Vienna, Austria URL; 2019. Available from: http://www.R-project.org/. Accessed July 3, 2020.

18. Fang C, Leavitt JA, Hodge DO, Holmes JM, Mohney BG, Chen JJ. Incidence and etiologies of acquired third nerve palsy using a population-based method. JAMA Ophthalmol. 2017;135(1):23. doi:10.1001/jamaophthalmol.2016.4456

19. Surachatkumtonekul T, Soontrapa P, Kampanartsanyakorn S, Dulayajinda D. Causes and treatment outcomes of third, fourth and sixth cranial nerve palsy. J Med Assoc Thai. 2012;95 Suppl 4:S96S101.

20. Sanders S, Kawasaki A, Purvin VA. Patterns of extraocular muscle weakness in vasculopathic pupil-sparing, incomplete third nerve palsy. J Neuro-Ophthalmol. 2001;21(4):256-259. doi:10.1097/ 00041327-200112000-00005

21. Dhume K, Paul K. Incidence of pupillary involvement, course of anisocoria and ophthalmoplegia in diabetic oculomotor nerve palsy. Indian J Ophthalmol. 2013;61(1):13. doi:10.4103/0301-4738.99999

22. Elmalem VI, Hudgins PA, Bruce BB, Newman NJ, Biousse V. Underdiagnosis of posterior communicating artery aneurysm in noninvasive brain vascular studies. J Neuro-Ophthalmol. 2011;31 (2):103-109. doi:10.1097/WNO.0b013e3181f8d985

23. Kupersmith MJ, Heller G, Cox TA. Magnetic resonance angiography and clinical evaluation of third nerve palsies and posterior communicating artery aneurysms. J Neurosurg. 2006;105(2):228-234. doi:10.3171/jns.2006.105.2.228

24. White PM, Wardlaw JM, Easton V. Can noninvasive imaging accurately depict intracranial aneurysms? A systematic review. Radiology. 2000;217(2):361-370. doi:10.1148/radiology.217.2.r00nv06361

25. White PM, Teasdale EM, Wardlaw JM, Easton V. Intracranial aneurysms: CT angiography and MR angiography for detection - prospective blinded comparison in a large patient cohort. Radiology. 2001;219(3):739-749. doi:10.1148/radiology.219.3.r01ma16739

26. Lu L, Zhang LJ, Poon CS, et al. Digital subtraction CT angiography for detection of intracranial aneurysms: comparison with threedimensional digital subtraction angiography. Radiology. 2012;262 (2):605-612. doi:10.1148/radiol.11110486

27. Mathew MRK, Teasdale E, McFadzean RM. Multidetector computed tomographic angiography in isolated third nerve palsy. Ophthalmology. 2008;115(8):1411-1415. doi:10.1016/j.ophtha.2007. 12.021

28. Kaufmann TJ, Huston J, Mandrekar JN, Schleck CD, Thielen KR, Kallmes DF. Complications of diagnostic cerebral angiography: evaluation of 19826 consecutive patients. Radiology. 2007;243(3):812819. doi:10.1148/radiol.2433060536
Eye and Brain

\section{Publish your work in this journal}

Eye and Brain is an international, peer-reviewed, open access journal focusing on clinical and experimental research in the field of neuroophthalmology. All aspects of patient care are addressed within the journal as well as basic research. Papers covering original research, basic science, clinical and epidemiological studies, reviews and

Submit your manuscript here: https://www.dovepress.com/eye-and-brain-journal evaluations, guidelines, expert opinion and commentary, case reports and extended reports are welcome. The manuscript management system is completely online and includes a very quick and fair peerreview system, which is all easy to use. Visit http://www.dovepress. com/testimonials.php to read real quotes from published authors. 